\title{
Reliability-based fatigue life estimation of shear riveted connections considering dependency of rivet hole failures
}

\author{
Davide Leonetti ${ }^{1, *}$, Johan Maljaars ${ }^{1,2}$, and H.H. (Bert) Snijder $^{1}$ \\ ${ }^{1}$ Eindhoven University of Technology, Department of the Built Environment, Eindhoven, the Netherlands \\ ${ }^{2} \mathrm{TNO}$, Delft, the Netherlands
}

\begin{abstract}
Standards and guidelines for the fatigue design of riveted connections make use of a stress range-endurance $(\mathrm{S}-\mathrm{N})$ curve based on the net section stress range regardless of the number and the position of the rivets. Almost all tests on which S-N curves are based, are performed with a minimum number of rivets. However, the number of rivets in a row is expected to increase the fail-safe behaviour of the connection, whereas the number of rows is supposed to decrease the theoretical stress concentration at the critical locations, and hence these aspects are not considered in the S-N curves. This paper presents a numerical model predicting the fatigue life of riveted connections by performing a system reliability analysis on a double cover plated riveted butt joint. The connection is considered in three geometries, with different number of rivets in a row and different number of rows. The stress state in the connection is evaluated using a finite element model in which the friction coefficient and the clamping force in the rivets are considered in a deterministic manner. The probability of failure is evaluated for the main plate, and fatigue failure is assumed to be originating at the sides of the rivet holes, the critical locations, or hot-spots. The notch stress approach is applied to assess the fatigue life, considered to be a stochastic quantity. Unlike other system reliability models available in the literature, the evaluation of the probability of failure takes into account the stochastic dependence between the failures at each critical location modelled as a parallel system, which means considering the change of the state of stress in the connection when a ligament between two rivets fails. A sensitivity study is performed to evaluate the effect of the pretension in the rivet and the friction coefficient on the fatigue life.
\end{abstract}

\section{Introduction}

Most of the steel bridge structures designed in the $19^{\text {th }}$ century until about the second half of the $20^{\text {th }}$ century were built by employing built-up structural members assembled by hot riveting, i.e. the manufacturing process of installing rivets by hotforming one of the heads, usually done in-situ. To date, a number of riveted bridges is still in service. Making available models and methods able to estimate the safety of the riveted connection with respect to fatigue is a topic of high relevance for these bridges [1].

Several variables have been shown to affect the fatigue performance of riveted structures. Extensive reviews are reported in $[2,3]$, among others. In case of a shear connection, there is experimental evidence that fatigue failure does not originate in the rivets but in the plate. This means that the fatigue life depends on features such as the relative size of the hole with respect to the plate, the method of hole forming, the bearing condition of the rivet with respect to the hole and the clamping force [4]. The clamping force provided by the rivet is due to the circumstance that when the rivet cools down it tends to reduce in length, but it is constrained by the plates, determining the grip length. The scatter shown in fatigue test data of riveted joints has been attributed not only to the variability of the fatigue properties within the material, but also to the clamping force that varies from rivet to rivet [5]. It has been observed that a higher clamping force induces a beneficial effect on the fatigue life [5][7]. Several investigations have been performed to evaluate the clamping force. It has been found to be dependent on the type of steel [8] and to increase with the grip length [8] [9]. In addition, it has been shown to be independent of the hot forming process [8], which can include manual or hydraulic hammering, but dependent on the hammering time and temperature [10]. Based on the few results reported in [8], the clamping force has been related to yield stress of the rivet material by evaluating the ratio between the nominal tensile stress in the rivet shank cross-section due to the clamping force, namely the clamping stress, and the measured yield stress of the rivet [11]. The analyses conducted report an average value of 0.70 . However, due to the dependence on the grip length, it has to be indicated that average values of 0.61 and 0.77 result 
for grip lengths of $75 \mathrm{~mm}$ and $125 \mathrm{~mm}$, respectively. In [12], based on a comparison between static load displacement diagrams and numerical simulations, a value of 0.5 is suggested as an optimal value.

The failure of a shear joint with multiple rivets in a row involves a complex response to fatigue loading. Because of the stochastic nature of fatigue, cracks nucleate at the critical locations not at the same time, even when the local stresses are equal. In addition, during crack propagation, both the local stiffness of the plate and the distribution of the load among the fasteners change. This process is known as multiple- site damage [3].

Recently, research has been done in order to assess the reliability of riveted structural details and bridge structures [13]. Based on the test data available in the literature, also new fatigue classes were proposed according to their proneness to fatigue failure [14]. Reliability models have been formulated to quantify the safety of both structural details [15] and bridges [16]. An extensive review of the method employed is reported in [17]. In [18] a probabilistic analysis was performed to estimate the probabilistic stress range vs endurance field ( $\mathrm{P}$ $\mathrm{S}-\mathrm{N}$ curves) of a riveted shear splice. In order to do so, the strain-life data related to the plain material have been employed in combination with the results of a finite element (FE) model by employing linear elastic material properties. In [19] a probabilistic approach has been presented to estimate the fatigue resistance and its scatter for a double shear riveted butt joint with one rivet per side. The material used was tested to determine the monotonic and cyclic static properties as well as the fatigue crack propagation data. The stress range vs endurance relation was simulated in a Monte Carlo analysis estimating the number of cycles to crack initiation and the subsequent propagation. The results were compared with fatigue test data showing a good agreement. In addition, the results confirm that a large portion of the fatigue life of the connection analysed is determined by the crack nucleation period. A system reliability model has been proposed in [15] in order to estimate the reliability of a cross-girder-to-stringer connection under variable amplitude fatigue loading. A FE model and the theory of critical distances have been used to estimate the fatigue life in each Monte Carlo sample. The model is based on the assumption that collapse due to fatigue may occur in several potential failure modes, resulting from the failure at specific hot-spot. The probability underlying each identified mode of failure can be estimated from the probabilities of failure evaluated at the hot-spots that contribute to it, which are assumed to be statistically independent. Although this assumption may be a good approximation for a particular type of connection, its use might determine a wrong estimation of the survival probability of the considered mode of failure for shear riveted connections. Indeed, after that a failure occurs at a hot-spot, the remaining hot-spots determining the same mode of failure will experience a more severe state of stress with respect to fatigue.

Fig. 1. Definition of the three geometries: 1) riveted double cover butt joint, 2) riveted double cover butt joint with two rivet rows, 3 ) riveted double cover butt joint with two rivets.

In this paper, the authors present a method to estimate the fatigue life distribution of a doublecovered butt riveted connection with multiple rivets
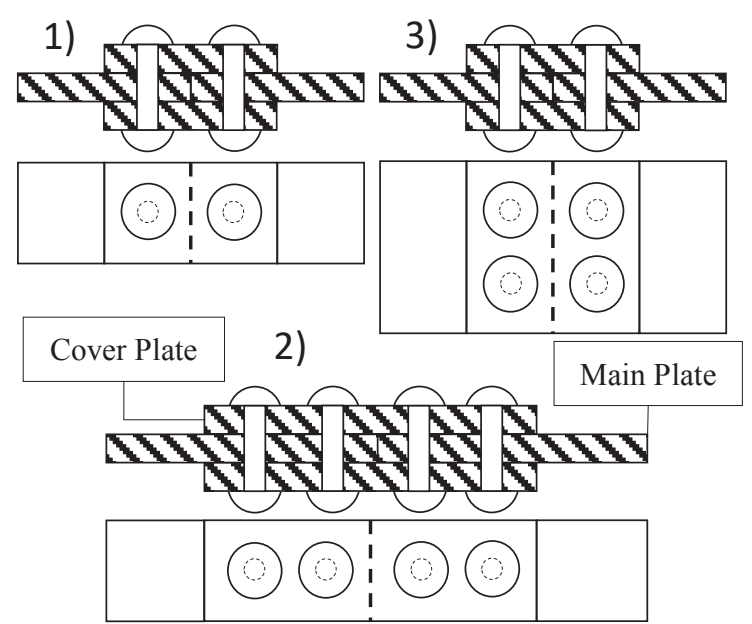

based on a system reliability model. The proposed formulation is able to consider statistical dependency between failures at different hot-spots, using a limited number of FE simulations, which results in modelling the multiple-site damage phenomenon. For the purpose of this study, three geometries have been defined as shown in Figure 1.

\section{Models and methods}

\subsection{Finite element model}

The FE model has been generated in ANSYS 17.2 for the purpose of evaluating the state of stress in the considered connection under the external loads. For each geometry, the FE model consists of the following bodies:

- The main plates.

- The cover plates.

- The rivets.

The diameter of the holes and the rivet shank are set equal to $20 \mathrm{~mm}$, the diameter of the rivet head is 30 $\mathrm{mm}$, the ratio between the gross and net section areas of the plates is 1.4 and the edge distance in the loading direction is $70 \mathrm{~mm}$. The thickness of the main plate is set to be equal to the thickness of each cover plate. For this reason, failure is expected to occur in either one of the main plates that are assumed to be independent with respect to their failure behaviour. If a crack is not present, the 
geometry is three times symmetric. When a ligament fails, only one plane of symmetry exists. Thus, half of the connection geometry is modelled to reduce the computational effort, see Figure 2. The element type employed to discretize the geometrical model is a 20-node isoparametric element (SOLID186).

Contacts and interaction between the plates and the rivets are simulated by applying contact and target elements, (CONTA174) and (TARGE170) respectively, further meshing the surfaces that might penetrate each other. The Augmented Lagrange formulation is used in order to simulate contact interaction. The Coulomb friction model is used to simulate the friction between plates and between the rivet and plate. The displacement vectors in the tangential direction at the two contact surfaces equal each other when the following

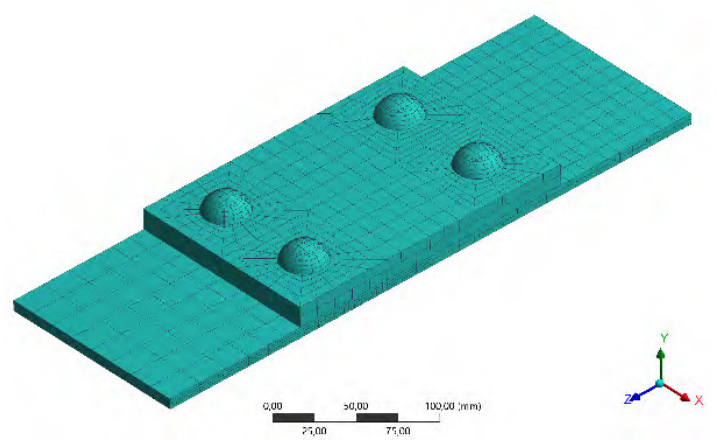

Fig. 2 FE model of half the connection.

condition occurs:

$$
\left\|t_{T}\right\|<\mu_{s} \boldsymbol{t}_{N}
$$

where $\left\|\boldsymbol{t}_{T}\right\|$ is the vector of the tangential stress evaluated at the contact nodes, $\mu_{s}$ is the static friction coefficient and $\boldsymbol{t}_{N}$ is the vector of the normal stresses evaluated at the contact nodes. Based on the values reported in [20], [21], the friction coefficient has been set equal to 0.33 . The rivet pretension has been implemented by placing a negative thermal load to the rivet shank. The value of the thermal load applied to the rivet is such that the average tensile stress in the rivet is $188 \mathrm{MPa}$, which is estimated by dividing the mean yield stress for a St44 steel grade, evaluated according to [22], and equal to $312 \mathrm{MPa}$ by 0.6 , according to the findings of $[8,11]$. The connection is then loaded by applying a far field pressure to the main plate. The thermal and pressure loads have been implemented in three "Steps":

1. The thermal load is applied (and hereafter kept constant);

2. The far field load is applied to the maximum value;

3. The far field load is reduced to zero.

The third step is performed to evaluate the effects of the load-sequence, which is expected since the model is non-linear.
A convergence study has been performed to find the mesh size, non-linear solution controls and the contact settings, i.e. the normal contact stiffness and allowable penetration, to be used in the analysis.

\subsection{Notch stress estimation}

The stress range to be used for the fatigue life estimation has been estimated by employing the FE method in conjunction with the gradient method in the FKM Guidelines [23], in order to consider both the influence of the stress concentration and the stress gradient on the fatigue life. In this method, the fatigue notch stress is defined as

$$
\begin{gathered}
\sigma=\sigma_{\text {nom }} K_{f} \\
K_{f}=K_{t} / n_{\sigma}
\end{gathered}
$$

where $K_{f}$ is the fatigue notch factor, $K_{t}$ is the theoretical stress concentration factor and $n_{\sigma}$ accounts for the stress gradient effect on the fatigue life. It depends on both the material, by the constants $a_{G}$ and $b_{G}$ and the ultimate tensile strength $\sigma_{u}[\mathrm{MPa}]$, and the stress gradient $G_{\sigma}$ $[\mathrm{MPa} / \mathrm{mm}]$ at the notch root, by the following:

$$
n_{\sigma}=\left\{\begin{array}{lc}
1+G_{\sigma} \cdot m m \cdot 10^{-\left(a_{G}-0.5+\frac{\sigma_{u}}{b_{G} \cdot M P a}\right)} & G_{\sigma} \leq 0.1 \\
1+\sqrt{G_{\sigma} \cdot m m} \cdot 10^{-\left(a_{G}+\frac{\sigma_{u}}{b_{G} \cdot M P a}\right)} & 0.1<G_{\sigma} \leq 1 \\
1+\sqrt[4]{G_{\sigma} \cdot m m} \cdot 10^{-\left(a_{G}+\frac{\sigma_{u}}{b_{G} \cdot M P a}\right)} & 1<G_{\sigma} \leq 100
\end{array}\right.
$$

where $a_{G}$ and $b_{G}$ are dimensionless material constants respectively equal to 0.50 and 2700 for non stainless steels. The maximum fatigue notch stress, $\sigma_{\max }$, and the minimum fatigue notch stress, $\sigma_{\text {min }}$, are evaluated at the maximum applied load $\left(P=P_{\max }\right)$ and the minimum applied load $(P=$ $\left.P_{\text {min }}\right)$, respectively. The mean stress $\left(\sigma_{m}^{*}\right)$, and the stress amplitude $\left(\sigma_{a}\right)$ at the notch can be evaluated. The mean stress is marked with an asterisk because it might be corrected in case elastic shakedown would occur. Indeed, when dealing with an elastic ideally plastic material, elastic shakedown occurs if the maximum notch stress exceeds the yield stress $\left(\sigma_{y}\right)$ and the related stress amplitude is smaller than the yield stress. This assumption is also realistic for steels, which exhibit a yield plateau. The corrected mean stress $\left(\sigma_{m}\right)$ is:

$$
\left\{\begin{array}{cc}
\sigma_{m}=\sigma_{y}-\sigma_{a} & \text { if } \sigma_{\text {max }}^{*}>\sigma_{y} \text { and } \sigma_{a}<\sigma_{y} \\
\sigma_{m}=\sigma_{m}^{*} & \text { if } \sigma_{\max }^{*} \leq \sigma_{y}
\end{array}\right.
$$

The mean stress and the stress amplitude characterizing the stress fluctuation at each hotspot location are then further modified by employing the Goodman mean stress correction model in order to evaluate the equivalent stress amplitude $\left(S_{a}=\right.$ $\Delta S / 2)$ at a stress ratio ( $\mathrm{R})$ equal to -1 : 


$$
S_{a}=\frac{\sigma_{a}}{1-\frac{\sigma_{m}}{\sigma_{u}}}
$$

\subsection{Fatigue resistance estimation}

In [22] a method to estimate the tensile strength properties of structural steels from the nominal yield stress is proposed based on an extensive analysis of test data available in literature. In particular, the ultimate tensile strength and the yield stress of low strength structural steels are found to be distributed following a Bivariate Normal Probability density function (Pdf). The mean values of the distribution, the correlation matrix and the coefficient of variation of the Pdf are reported in [22]. According to [22], the mean yield stress and the mean ultimate tensile strength for St52 steel grade are $409 \mathrm{MPa}$ and $613 \mathrm{MPa}$, respectively. The stress range vs. endurance relation is formalized by employing the Basquin relation

$$
\log _{10} N=\log _{10} C_{0}+m \log _{10}(\Delta S)
$$

where, $\log _{10} \mathrm{C}_{0}$ and $\mathrm{m}$ can be derived based on an estimation of the fatigue limit $\left(\Delta S_{0}\right)$, the fatigue resistance at two million cycles, and the fatigue strength coefficient $\left(\sigma_{f}^{\prime}\right)$, the intercept of the elastic S-N curve with the stress range axis, as follows:

$$
\begin{gathered}
m=\frac{\log _{10} N_{0}}{\log _{10}\left(\Delta S_{0}\right)-\log _{10}\left(2 \sigma_{f}^{\prime}\right)} \\
\log _{10} C_{0}=\log _{10} N_{0}-m \log _{10}\left(\Delta S_{0}\right)
\end{gathered}
$$

The fatigue limit is determined by making use of the relation prosed in [24], where the stress amplitude $S_{0}$ determining the fatigue limit at $N_{0}=2$ million cycles and $\mathrm{R}=-1$ is related to the ultimate tensile strength by:

$$
S_{0}=0.5 N(1,0.15) \sigma_{u} k_{a}
$$

Where $\mathrm{N}$ denotes a Normal distribution with average and standard deviation equal to 1 and 0.15 , respectively. The surface factor $\left(k_{a}\right)$ is here employed in order to take surface finishing into account. The following relation, valid for machined surfaces, is considered [24]:

$$
k_{a}=4.45 \sigma_{u}^{-0.2653} \operatorname{LN}(1,0.060)
$$

Where the second term of the equation is a lognormal distribution with average and standard deviation equal to 1 and 0.06 , respectively. The evaluated fatigue surface factor, $k_{a}$, also takes into account the variability of the surface finishing. The mean and the standard deviation of the fatigue strength coefficient are estimated by employing the relations reported in [25], [26] for carbon steels:

$$
E\left(\sigma_{f}^{\prime}\right)=0.95 E\left(\sigma_{u}\right)+400
$$

$$
\operatorname{std}\left(\sigma_{f}^{\prime}\right)=0.95 \frac{\operatorname{std}\left(\sigma_{u}\right)}{\rho_{\sigma_{u}, \sigma_{f}^{\prime}}}
$$

where $\rho_{\sigma_{u}, \sigma_{f}^{\prime}}$ is the correlation coefficient, which is reported to be equal to 0.8 , and $\mathrm{E}(\cdot)$ is the expectation operator. In the framework of this paper, the mean value of the ultimate tensile strength and the yield stress are assigned to each hotspot. Then, to estimate the fatigue variability at each critical location, Equations (8)-(13) have been employed.
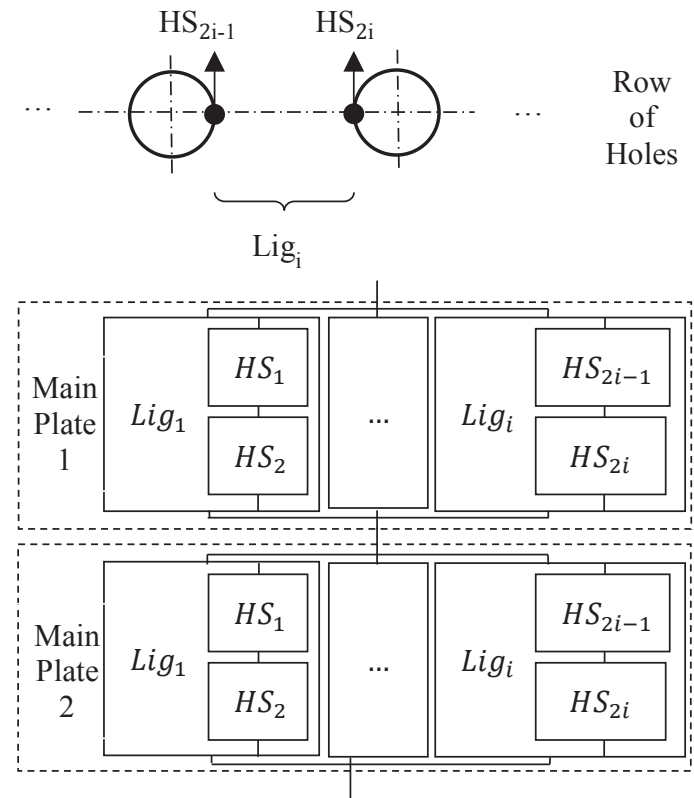

Fig. 3 Relation between ligament and hot spot location for a row of holes and box diagram showing the system reliability model employed to describe the failure in each main plate.

\subsection{System reliability modelling}

To evaluate the fatigue life of the selected geometries a system reliability model should be set. Given the considered geometry, the fatigue life is determined by the failure of either one of the main plates.

In general, the connection consists of a number of components and thus different failure modes might be expected, each one of these determined by failure at one or more hot-spots. Thus, since the occurrence of any of the failure modes determines the failure of the connection, the connection is considered as a series system of the failure modes. In this paper, since only one failure mode is considered, the probability that the system fails equals the probability that failure occurs in the considered failure mode. However, it is recognized that other modes of failure might exist.

According to Figure 3, the considered mode of failure is assumed to take place when all the ligaments in one of the two main plates have failed. 
Whit respect to one main plate, its failure can be assumed as a parallel system:

$$
\operatorname{Pr}\{\text { Mode }\}=\operatorname{Pr}\left\{\operatorname{Lig}_{1} \cap \ldots \cap \operatorname{Lig}_{i}\right\}
$$

The failure of a ligament is directly associated to the failure at either one of the hot-spots related to it, see Figure 3. This means that the probability that a ligament fails is equal to:

$$
\operatorname{Pr}\left\{\operatorname{Lig}_{i}\right\}=\max \left[\operatorname{Pr}\left\{H S_{2 i-1}\right\} ; \operatorname{Pr}\left\{H S_{2 i}\right\}\right]
$$

since the failure events at the hotspots are fully correlated. The full correlation is due to the fact that if one of the two hotspot fails also the ligament fails, which automatically means failure at the other hot-spot, see Figure 3.

\subsection{Solution of the reliability model}

The Monte Carlo method is employed to evaluate the probability of failure of the considered mode at a certain stress range value. The FE model described in section 2.1 is used to determine the stress state in the connection in any possible combination of ligament failure that does not cause static failure of the main plate at $\mathrm{P}=\mathrm{P}_{\max }$, or plastic shakedown under cyclic loading. At each stress range the simulated dataset of $k=10000$ samples has been produced by employing the following algorithm:

a) Based on the selected geometry a FE model is created to evaluate the state of stress in the connection for the identified failure scenarios of interest.

b) The value of the stress range to be applied to the far field cross-section is chose together with the stress ratio $R$. The FE model is solved.

c) At each hot-spot, the maximum principal stresses are extracted from the FE model at $\mathrm{P}=\mathrm{P}_{\max }$ and $\mathrm{P}=\mathrm{P}_{\text {min }}$.

d) The stress values are corrected using Equations (4)-(6) to estimate the equivalent stress amplitude for a fully reversed cyclic loading.

e) At each hot-spot, the following are assigned:

- The values of the monotonic strength properties, which are the same at each hotspot;

- The parameters of the Basquin relation, by randomly sampling a value of the fatigue limit and the fatigue strength coefficient;

- The value of the cumulative, the critical and the residual Palmgren-Miner damage;

- The hot-spot status, which is "not failed" at the first iteration, and can be "failed" in a later stage.

f) A state parameter storing the calculated number of cycles to failure for the entire connection is initialized for the current $k$-th sample. g) For each hot-spot, the predicted number of cycles to failure is determined. The minimum non-zero value obtained represents the number of cycles to the next failure at a certain hotspot. Based on this value, the partial damage due to the current iteration is calculated for each hot-spot location. The cumulated damage, the cumulated number of cycles and the failure status are updated at each critical location.

h) The damage at a certain hot-spot exceeds the critical damage, and the corresponding ligament is assumed to be cracked, determining the failure scenario of interest in the next iteration. Thus, based on the results of the current iteration, the failed critical location is determined as well as the failed ligament. Thus, the stress state belonging to this new configuration is evaluated and employed in the next iteration.

i) The steps from g) to i) are repeated until either plastic shakedown occurs $\left(\sigma_{a}>\sigma_{y}\right.$, see Equation (5)), or the remaining net-section is no longer able to carry the maximum applied load.

j) The steps from e) to j) are repeated until the total number of samples is reached.

The steps from a) to d) have been performed in ANSYS Workbench environment. Then, the results have been exported to be processed in MatLab for the following steps from e) to $\mathrm{k}$ ).

\section{Results and discussion}

Geometries 1 and 2 are compared in terms of S-N curves in which the nominal net section stress range is plot against the number of cycles to failure, assumed to occur when a hot-spot fails.

In order to compare geometries 1 and 3, the results are expressed in terms of cumulative distribution functions (Cdf) of the fatigue life at a given net stress range. For the analysis, two arbitrary net section stress ranges have been selected, $140 \mathrm{MPa}$ and $161 \mathrm{MPa}$, considering a zero pulsating stress cycle, i.e. stress ratio $R=0$.

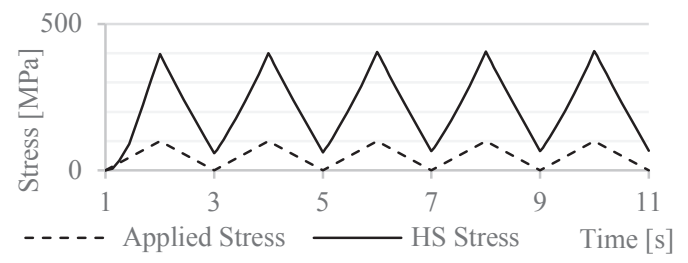

Fig. 4 Applied stress and principal Stress at the hotspot as function of the load step.

\subsection{Geometry 1}

When the external load drops to zero after reaching the maximum load in the first cycle, a residual state of stress remains in the main plate. This phenomenon is found to stabilize after the first 
cycle, as shown in Figure 4 where five constant amplitude stress cycles $(\Delta \sigma=100 \mathrm{MPa}, \mathrm{R}=0)$ have been applied at the gross section and the corresponding stress at the hot-spot has been plot. This can be addressed to the nonlinearities introduced by slipping at the contacting surface. Despite at macro level the friction condition $\left(P<\mu F_{c}\right)$ may be satisfied and slipping should not occur, in the vicinity of highly stressed locations, the friction condition imposed by Equation (1) might not be satisfied. This results into local slipping that is not recovered during the unloading phase. Thus, a load sequence effect occurs. Each critical location is subjected to a fatigue loading characterized by both stress concentration and a stress ratio higher than the nominal one. In addition, when the contact is not able to carry the load by friction, the rivet carries part of the total load by shear, so the higher the maximum net section stress applied, the higher is the theoretical stress concentration factor $\left(K_{t}\right)$ and thus, the fatigue notch factor $\left(K_{f}\right)$. At a relatively low far field stress range level, the load is transferred mainly by friction having smaller stress concentration at the hotspot. The results, in terms of nominal S-N curve, in which the stress range refers to the net section, are plot in Figure 5 where a comparison is made with the $\mathrm{S}-\mathrm{N}$ curve of the parent material for $\mathrm{R}=-1$. In the finite life region, the median fatigue life is directly obtained by the Monte Carlo samples resulting from the reliability model for each selected stress ranges. The median fatigue limit has been estimated from the same Monte Carlo samples by employing a Probit analysis: at each stress range level, the ratio between failed and non-failed simulations has been interpolated by a normal, lognormal and generalized extreme value Cdf using the least square method in order to estimate the parameters of the distribution. Based on the coefficient of determination, the generalized

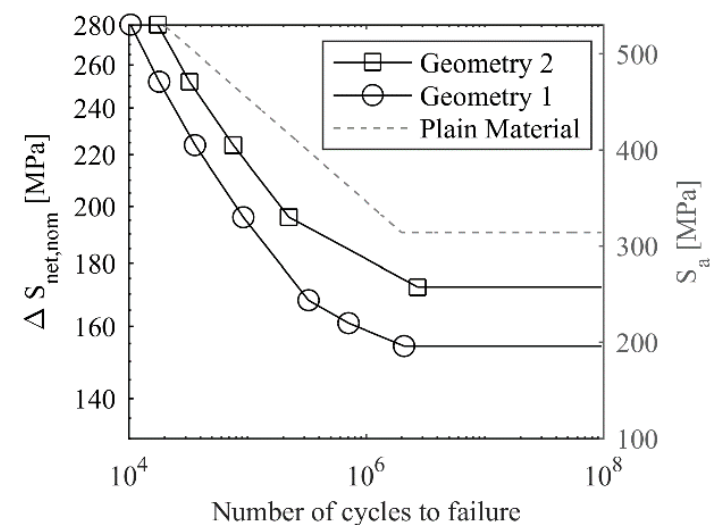

Fig. 5 Median S-N curves resulting from the analysis for geometry 1 and 2 . The continuous line is the S-N curve obtained for geometry 1 and 2 by employing the the estimated S-N curve of the plain material (dashed line), which stress amplitudes are reported in the secondary $\mathrm{y}$-axis for an alternating stress fluctuation $(R=-1)$. extreme value distribution has been selected and employed to estimate the median value of the fatigue limit (154 MPa).

At high far field stress ranges, both the stress concentration and the stress ratio at the hotspot increase and consequently, the resulting S-N curve tends to be steeper than the S-N curve of the plain material. At low stress range levels, the S-N curve becomes shallower and then approaches the fatigue limit. This is in line with the findings of [14] in terms of having a stepwise S-N curve describing the fatigue life of shear riveted connections.

\subsection{Geometry 2}

For geometry 2, the generalized extreme value distribution has been selected to estimate the fatigue limit. The estimated median value of the fatigue limit is $172 \mathrm{MPa}$. As it is shown in Figure 5, at the same net section stress range level the fatigue life of geometry 2 is higher compared to geometry 1. This because the maximum principal stress resulting from the $\mathrm{FE}$ analysis at the most stressed critical location is lower for geometry 2 as compared to geometry 1. Moreover, a higher percentage of the total load is transferred by friction and, eventually, transferred by bearing of the second rivet. Hence, it is advantageous to have multiple rows of rivets.

\subsection{Geometry 3}

At the hot-spot locations, the maximum principal stresses obtained by the FE model for this case are not significantly different from those obtained for geometry 1. Despite this, the first ligament failure occurs on average at a lower number of cycles. This is caused by the fact that first failure occurs at the weakest hot-spot. Thus with respect to the first failure, the higher the number of rivets in a row, the higher is the number of the hot-spots, the lower is the average life to first failure and the more this distribution is skewed towards lower life. On the other hand, the fail-safety of the connection is higher than for geometry 1 , because the connection has some residual fatigue life after failure of the first ligament. This aspect determines a longer detection period with respect to the total life of the joint, meaning higher probability of detecting the damage. The higher is the applied stress range, the lower is the contribution of the residual life after failure of the first ligament to the total fatigue life, see Figure 6. According to these findings, when considering the entire joint, failure of the first ligament occurs earlier than in the geometry 1 but, since there is more redundancy, the additional life associated with failure of the entire joint after failure of the first ligament depends on the stress range level. 


\subsection{Sensitivity study}

For geometry 1, a variation of the clamping force and the friction coefficient is considered and the maximum and minimum principal stresses at the hot-spots are calculated for a far-field stress range of $100 \mathrm{MPa}$ at $R=0$. The friction coefficient is assumed to

vary between 0.1 and 0.6 whereas the nominal stress in the rivet due to the clamping force is assumed to vary between zero and the nominal yield stress of the rivet material, which is $275 \mathrm{MPa}$. The results are expressed in terms of maximum principal stress, fatigue notch stress range and stress ratio and are shown in Figure 7. In any case, the theoretical stress concentration factor $\left(K_{t}\right)$ ranges between 2 and 5. It results that at low clamping, $K_{t}$ is almost independent of the friction coefficient and is close to 5. It is clear that the lower the friction coefficient, the less $K_{t}$ depends on the clamping. At relatively low clamping and low friction values, no residual stress is present at the hot-spots. At relatively high clamping and high friction values, when the load is mainly transferred by friction, the stress after unloading also tends to approach a minimum. In the region of interest, when $0.2<$ $\mu<0.4$ and the clamping stress is about $0.5-0.7$ times the yield stress, the higher stress ratios occur $(>0.3)$ and the stress ranges are in the order of 250$400 \mathrm{MPa}$. The obtained variability suggests that the estimated fatigue life is strongly dependent on the friction coefficient and the clamping stress, confirming the findings in [4]. Moreover, the analysis shows that the phenomena involved determine a complex response to loading and unloading, making it difficult for the double cover riveted butt joint to formulate any "rule of thumb".
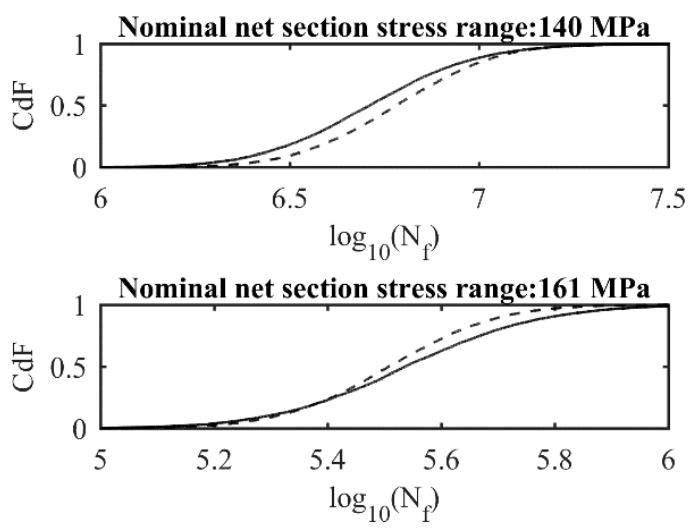

Fig. 6 Cumulative distribution of the number of cycles to failure for geometries 1 (continuous) and 3 (dashed).

\section{Conclusions}

The position and the number of the rivets have been shown to affect the state of stress in the connection under external loading. In order to evaluate the fatigue life of shear riveted connections, a reliability model and an algorithm to solve it have been proposed in the present paper.

The results highlight that the fatigue life increases with increasing number of rivet rows. By increasing number of rivets in a row, the fail-safety of the connection increases. However, this does not necessarily mean a higher fatigue life since the number of cycles to first ligament failure is found to be decreasing with the increasing number of rivets in a row.

It is recognized that the S-N curve employed in guidelines for fatigue design of riveted connections, as [27], does not address the effect of the rivet number and position on the fatigue life. This because the number and the position of the rivets employed in the specimens used to carry out fatigue tests significantly differs from the details often employed in practice. 
The formulated FE model estimates that high stress levels cause higher stress ratios and stress concentration factors than low stress levels. This justifies the findings reported in [14] of a two-slope $\mathrm{S}-\mathrm{N}$ curve to represent the fatigue life of shear riveted connections.

In accordance with the findings reported in [4]-[7], the fatigue life and stress state at the hotspot location strongly depend on the friction coefficient of the contacting surfaces and on the clamping force exhibited by the rivet. Further investigation is needed to quantify and predict these aspects. Further development is also needed in order to increase the accuracy of the estimation of the probabilistic stress range vs. endurance field. In particular, the presented algorithm does not consider the uncertainties related to the friction coefficient and the clamping force. In addition, since the use of the stress approach is limited to elastic shakedown, employing the notch strain approach would increase the accuracy and the domain of application of the fatigue life estimation.

The authors would like to acknowledge the Dutch infrastructural asset owners Rijkswaterstaat and ProRail and the research organization TNO for their support.

\section{References}

[1] K. A. Baker and G. L. Kulak, "Fatigue of riveted connections," Can. J. Civ. Eng., vol. 12, no. 1, pp. 184-191, 1984.

[2] G. L. Kulak, J. W. Fisher, and J. H. A. Struik, Guide to Design Criteria for Bolted and Riverted Joints. 2001.

[3] G. T. Hahn and C. A. Rubin, Structural shear joints analyses, properties and design for repeat loading. ASME Press, 2005.

[4] J. D. DiBattista, D. E. J. Adamson, and G. L. Kulak, "Fatigue Strength of Riveted Connections," J. Struct. Eng., vol. 124, no. 7, pp. 792-797, Jul. 1998.

[5] J. W. Carter, "Fatigue in Riveted and Bolted Single Lap JointsTitle," Proc. Am. Soc. Civ. Eng., vol. 80, no. 8, pp. 1-35, 1954.

[6] J. F. Parola, E. Chesson, Jr., and W. H. Munse, "Effect of bearing pressure on fatigue strength of riveted connections," 1965.

[7] W. H. Munse and E. Chesson Jr, "Riveted and bolted joints: net section design," $J$. Struct. Div., vol. 89, no. 1, pp. 107-126, 1963.

[8] W. M. Wilson and F. P. Thomas, "Fatigue Tests of Riveted Joints," no. 79, pp. 173$195,1938$.

[9] F. Baron and E. W. Larson, "High-Strength Bolts in Structural Joints: A Symposium: Comparison of Bolted and Riveted Joints,"
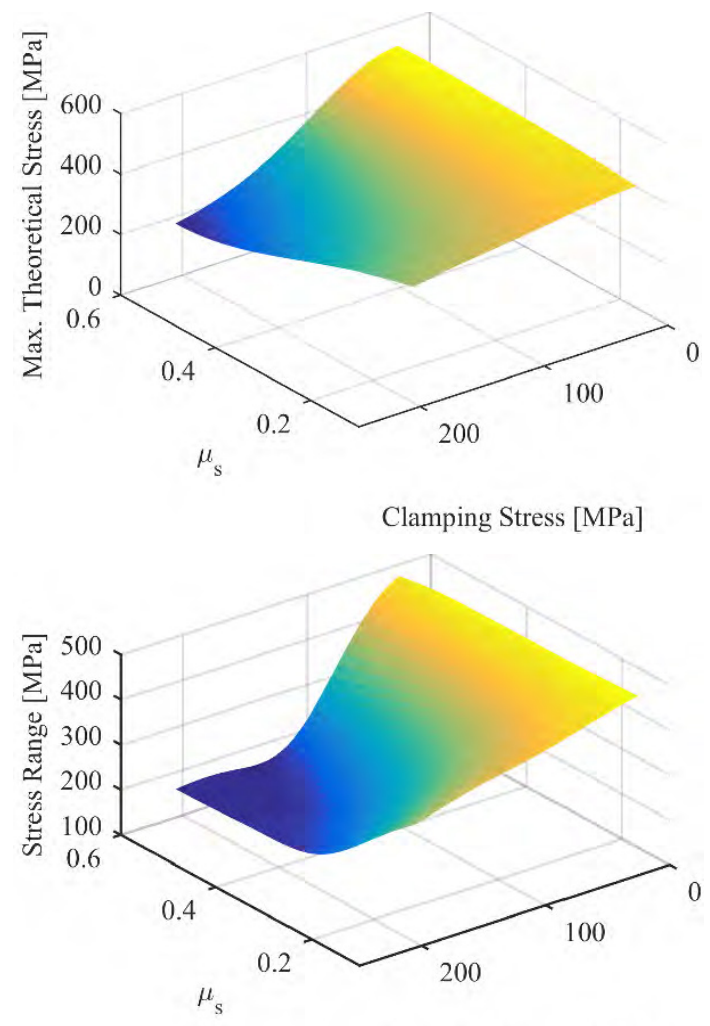

Clamping Stress [MPa]

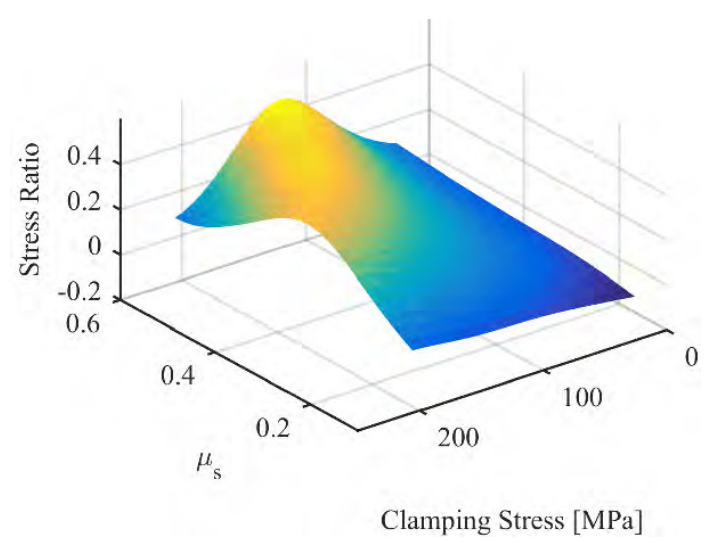

Fig. 7. Maximum theoretical stress, stress range and stress ratio evaluated at a critical location as function of the friction coefficient and the clamping for a stress range of $100 \mathrm{MPa}$ applied to the gross section.

Trans. Am. Soc. Civ. Eng., vol. 120, no. 1, pp. 1322-1334, 1955.

[10] O. Graf, Versuche mit nietverbindungen. J. Springer, 1941.

[11] B. Åkesson, "Fatigue Life of Riveted Railway Bridges," p. 169, 1994.

[12] R. Marmo, "Numerical and experimental investigation on the shear behaviour of riveted connections," 2011.

[13] C. Cremona, B. Eichler, B. Johansson, and T. Larsson, "Improved Assessment Methods for Static and Fatigue Resistance of Old Metallic Railway Bridges," J. Bridg. Eng., vol. 18, no. 11, pp. 1164-1173, 2013.

[14] A. Taras and R. Greiner, "Development and 
Application of a Fatigue Class Catalogue for Riveted Bridge Components," Struct. Eng. Int., vol. 20, no. 1, pp. 91-103, Feb. 2010.

[15] B. M. Imam, M. K. Chryssanthopoulos, and D. M. Frangopol, "Fatigue system reliability analysis of riveted railway bridge connections," Struct. Infrastruct. Eng., vol. 8, no. 10, pp. 967-984, 2012.

[16] C. S. Wang, Y. Xu, A. R. Chen, and W. Z. Chen, "System Fatigue Damage Reliability Assessment of Railway Riveted Bridges," Key Eng. Mater., vol. 347, pp. 173-178, 2007.

[17] X. W. Ye, Y. H. Su, and J. P. Han, "A state-of-the-art review on fatigue life assessment of steel bridges," Mathematical Problems in Engineering, vol. 2014. Hindawi, pp. 1-13, 10-Feb-2014.

[18] A. M. P. De Jesus, H. Pinto, A. FernándezCanteli, E. Castillo, and J. A. F. O. Correia, "Fatigue assessment of a riveted shear splice based on a probabilistic model," Int. J. Fatigue, vol. 32, no. 2, pp. 453-462, Feb. 2010.

[19] R. F. Sanches, A. M. P. De Jesus, J. A. F. O. Correia, A. L. L. Da Silva, and A. A. Fernandes, "A probabilistic fatigue approach for riveted joints using Monte Carlo simulation," J. Constr. Steel Res., vol. 110, pp. 149-162, 2015.

[20] G. L. Kulak, J. W. Fisher, and J. H. A. Struik, Guide to design criteria for bolted and riveted joints, vol. 15, no. 1. 1988.

[21] M. Veljkovic et al., "High-strength tower in steel for wind turbines (Histwin)," 2009.

[22] JCSS, "Probabilistic Model Code - Part 3," Jt. Comm. Struct. Saf., 2000.

[23] B. Hänel, E. Haibach, T. Seeger, G. Wirthgen, and H. Zenner, "Analytical strength assessment of components in mechanical engineering." Forschungskuratoirium Maschinenbau VDMA Verlag, Frankfurt am Main, 2012.

[24] C. R. Mischke, "Prediction of Stochastic Endurance Strength," J. Vib. Acoust. Stress Reliab. Des., vol. 109, no. 1, p. 113, Jan. 1987.

[25] S. K. Park and F. V Lawrence, "Monte Carlo Simulation of Weldment Fatigue Strength," J. Constr. Steel Res., vol. 12, pp. 279-299, 1989.

[26] J. C. McMahon and F. V. Lawrence, "Predicting fatigue properties through hardness measurements," 1984.

[27] B. Kühn, M. Lukic, A. Nussbaumer, H. P. Günther, and R. Helmerich, "Assessment of Existing Steel Structures: Recommendations for Estimation of Remaining Fatigue Life," Aachen, Delft 